\title{
Measuring Orbital Angular Momentum (OAM) and Torque Transfer from Polarization Vortices with the Electron Microscopy Pixel Array Detector
}

Kayla X Nguyen ${ }^{1}$, Yi Jiang ${ }^{2}$, Michael C. Cao ${ }^{1}$, Prafull Purohit ${ }^{6}$, Ajay K. Yadav ${ }^{3}$, Javier Junquera ${ }^{4}$, Mark W. Tate ${ }^{6}$, Ramamoorthy Ramesh ${ }^{3}$, Sol M. Gruner ${ }^{2,5,6,7}$, and David A. Muller ${ }^{1,5}$

${ }^{1}$ School of Applied and Engineering Physics, Cornell University, Ithaca, NY, USA

2. Department of Physics, Cornell University, Ithaca, NY, USA

${ }^{3}$.Department of Material Science and Engineering, University of California, Berkeley

${ }^{4}$.Department of Physics, University of Cantaberia, Spain

${ }^{5}$ Kavli Institute at Cornell for Nanoscale Science, Ithaca, NY USA

${ }^{6 .}$ Laboratory of Atomic and Solid State Physics, Cornell University, Ithaca, NY, USA

${ }^{7}$ Cornell High Energy Synchrotron Source (CHESS), Cornell University, Ithaca, NY, USA

Ferroelectric polarization vortices realized in multi-layered complex oxides superlattices such as PbTiO3/SrTiO3[1] play an analogous role to magnetic topological structures, except encoded in the polarization field. Here orbital angular momentum (OAM) functions as a toroidal order parameter in the polarization vortex arrays that could form the basis for memory storage and devices. Traditionally, electron microscopy methods for probing OAM have produced angular momentum dependent beams in the form of vortex beams [2][3], where the electron probe is divided into three separate beams each with angular momentum, $+l, 0$, and $l$, respectively. However, because of the division in intensity, signal to noise remains low. Here, we demonstrate a new phase-sensitive detection method with high contrast for measuring the OAM of an electron beam; shape and resolution are not compromised. This novel phase imaging method for OAM is realized due to a resurgence of high-speed, momentum-resolved electron microscope detectors highlighted here by the electron microscopy pixel array detector (EMPAD) [4].

Using the EMPAD, we show how OAM of the scattered beam can be recovered to reconstruct the torque transfer from a polarization vortex lattice. In addition, this technique can be applied to other topological structures such as skyrmions and spin torque transfer electronics.

At single electron sensitivity, the EMPAD records the full convergent beam electron diffraction (CBED) pattern at $0.86 \mathrm{~ms} /$ frame with a full well of $>10^{6}$ primary electrons per pixel per image. To extract OAM using our methodology, the gradient specimen potential must first be reconstructed from the polarization vortex fields using the probability current flow $\langle P x\rangle$ and $\langle P y\rangle$. It is then related back to Ehrenfest's theorem where values from torque transferred are extracted (Fig. 1). Multislice simulation of 6x6 $\mathrm{PbTiO}_{3} / \mathrm{SrTiO}_{3}$ is used to test the robustness of our technique with direct comparison to OAM calculated from angular changes observed in the full wave function (Fig.2). Here, we obtained excellent correlation between the two techniques (Fig $2 \mathrm{c}, \mathrm{d}$ ). When applied to experimental data, overall measurements extracted for OAM can range over five orders of magnitude in length scales, making it optimal for measuring polarization fields and torque transfer in complex, extended patterns. Although this imaging method should work equally well for electric and magnetic structures, we expect this technique is particularly well suited for imaging the toroidal order parameter of ferroelectric polarization vortex arrays of $\mathrm{PbTiO}_{3} / \mathrm{SrTiO}_{3}$ superlattices [5]. 


\section{References:}

[1] Yadav, A. K. et al, Nature 530 (2016), p. 198.

[2] Uchida, M. et al, Nature 464 (2010), p. 737.

[3] Verbeeck, J. et al, Nature 467 (2010), p. 301.

[4] Tate W. M. et al, Microscopy and Microanalysis. 22 (2016), p. 237.

[5] Supported by NSF MRSEC program (DMR 1120296), Kavli Institute at Cornell.



Figure 1. Analysis of (a) polarization vortices from [200] and [020] diffracted disks using (b) CBED pattern. Vortices reconstructed from purple boxed region of (c) ADF, using (d) $\left\langle\mathrm{P}_{\mathrm{x}}\right\rangle$ and $\left\langle\mathrm{P}_{\mathrm{y}}\right\rangle$.

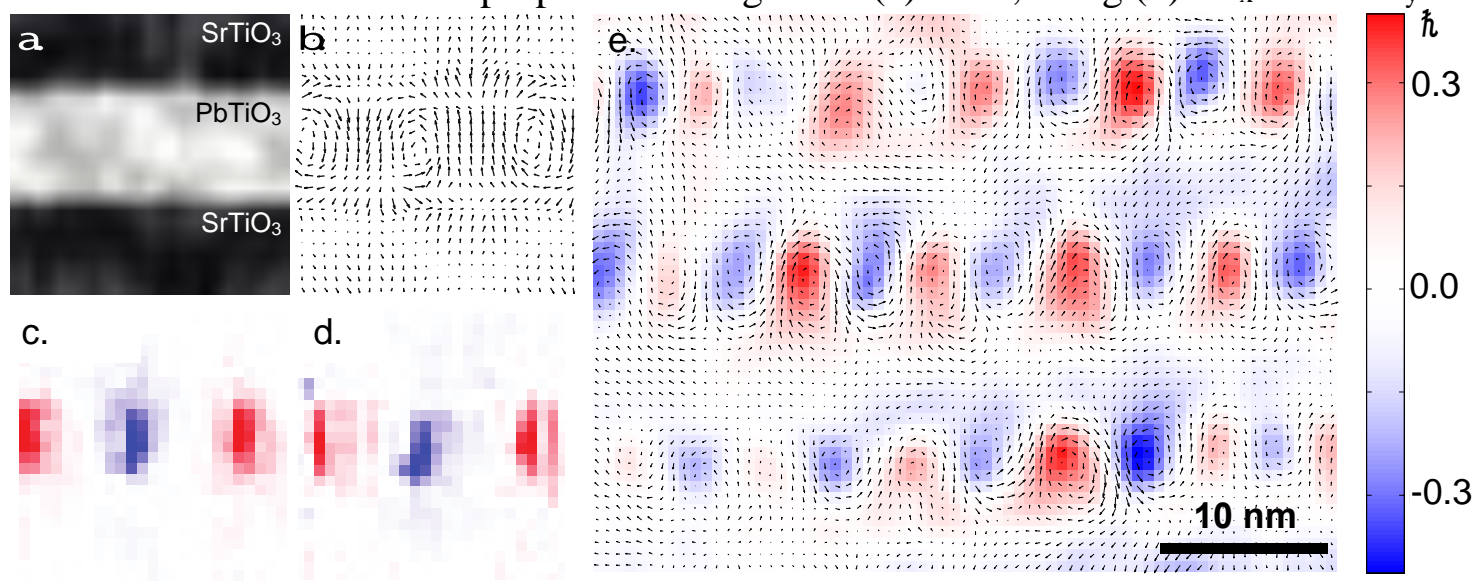

Figure 2. Multislice simulation for $6 \times 6 \quad \mathrm{PbTiO}_{3} / \mathrm{SrTiO}_{3}$ superlattice where (a) ADF image of $\mathrm{PbTiO}_{3} / \mathrm{SrTiO}_{3}$ is shown and (b) $\langle\mathrm{Px}\rangle$ and $\langle\mathrm{Py}\rangle$ extracted from simulation are used to reconstruct polarization vortices. Using the full wave function, OAM is observed by directly measuring angular changes in the wave function in (c), whereas the gradient of the potential reconstructed from the probability current flow is used to measure OAM in (d). Applied to experimental measurements, (e) transfer measurements extracted from orange boxed region in Fig. 1 c. Scale bar under (d) is $2 \mathrm{~nm}$. 